5. Salmon: Relation of Bovine Tuberculosis to the Public Health. Bulletin 33, Bureau of Animal Industry, p. 19 .

6. Same, pp. 16-19.

7. Ravenel : Paper Read before Pathological Society of Phila8 . Apres 24.1902 Univ. of Penna. Med. Bul., Mas, 1902, p. 66. ogenic Bacteria. Journal of l3oston Society of Medical Sciences. Jan. 16, 1900 , vol. iv, No. 5 , p. 102 .

9. Lartigau: Journal of Medical Research, July, 1901.

10. Ravenel: The Comparative Virulence of the Tubercle Bacillus from Human and Bovine Sources. Read before the British Congress on Tuberculosis, London, July, 1901. Univ, of Penna. Med. Bul. September, 1901 .

11. Dubard : Congrès pour l'étude de la tuberculose chez l'homme

et chez les animaux, 4th session, 1898, p. 711. (Table copied in Bul. 33,
12. Thorne: Harben Lectures, 1898. (The Bureau of Animal Industry, p. 23.)

13. Bottstein: Statistische Beitrïge zur Verbreitung der Tuberculose. München. Med. Wochenschr., Bd. 48 (41), Oct. 8, 1901, pp. $1610-1612$.

14. Report of Storrs (Conn.) Agricultural Experiment Station, 1898 , p. 11.

\section{THE INTERTRANSMISSIBILITY OF HUMAN AND BOVINE TUBERCULOSIS;}

A Review of THE EXPerimental EVIDENCE.*

\section{R. R. DINWIDDIE, M.D.}

FAYETTEVILLE, ARK.

Within the past two years a number of articles have appeared beside that of to-day more or less exhaustively reviewing the evidence as to the frequency or possibility of human tuberculosis originating by contagion from cattle. Such evidence as we can obtain must necessarily be circumstantial. Even if it were permissible to make direct inoculation or infection experiments on the human being, a settlement of the question could only be obtained by a series of comparative tests on many individuals with tubercle bacilli of human and bovine origin respectively, and accompanied by all the safeguards to exact experimentation which have been found necessary in our similar tests made on cattle. We can therefore see the folly and the futility of those oversanguine experimenters who propose to decide the matter by one clumsily conducted inoculation test on a human victim. Truly, "Fools rush in where angels fear to tread."

Beside the experimental evidence-along with which I include that which is derived from a comparison of the biologic characters of the bacilli-we may obtain some information from other sources: from a comparative study of the naturally acquired disease in man and animal in reference to its contributory causes, its course, its anatomic and histologic features, and from the records of accidental inoculation or supposed ingestion infection of human beings from bovine tubercular products. This part of the subject having been already discussed I shall limit myself to a few remarks on certain points only which have come under my own observation.

$$
\text { CONTRIBUTORY CAUSES. }
$$

With the exception of certain minor influences which are not in operation in the lower animals, contributory causes are closely comparable in the human and bovine animals. In a recent paper ${ }^{1} \mathrm{I}$ have attempted to show that in human as in bovine tuberculosis by far the most important of these so-called auxiliary causes is the universally prevalent disregard of adequate ventilation requirements in sleeping rooms and stables. Above all other conditions tuberculosis is associated with overcrowding-among cattle practically confined to stabled herds; in human beings the special scourge of the tenement houses, infrequent or even unknown among races of primitive habits yet exempt from the insanitary vices of our modern civilization.

* Read at the Fifty-third Annual Meeting of the American Medical Association, in the Section on Hygiene and Sanitary Science, and approved for publication by the Executive Committee: Drs. Arthur R. Reynolds, George Cook and Heman Spalding.
The course of the disease in cattle is not dissimilar from that of man. It is, as a rule, chronic, phthisis or pining, only showing itself as a late symptom. The number of animals showing any symptoms is only a small percentage of those actually infected. Recovery of individual cases under favorable conditions no doubt does occur, but on this point we have little knowledge. Latency or non-progressiveness appears to be as common a feature as in mankind.

\section{THE BOVINE ANATOMIC LESIONS.}

The anatomic lesions of the bovine disease are characterized by their predilection for the serous membranes, the magnitude of the tubercle conglomerates, their fibrous structure and tendency to calcareous deposits, and finally by the relative scarcity of tubercle bacilli. Ulcerative lung lesions are less common than in man, and for this reason the bronchial discharges are relatively less abundant and poorer in their content of tubercle bacilli. When pulmonary cavities or vomicæ do occur in cattle, the discharge is as profuse and the numbers of tubercle bacilli as great as in advanced pulmonary phthisis of man. Moreover, the tubercle bacllli present in such specimens do not differ morphologically from those of human sputa. These facts I have been able to demonstrate from specimens of ulcerative lung disease in cattle obtained many years ago, and now on exhibition here. In regions not open to external infection, as in lymph nodes and on serous membranes, the necrotic tissues do not liquefy and become purulent, but appear either as tough coherent masses or as accumulations of unorganized debris of butter-like consistency in which tubercle bacilli are usually difficult of demonstration. Specimens illustrating these conditions may be seen in all museum collections of cattle tuberculosis.

In the records of accidental infection of mankind by bovine tubercular products either by inoculation or supposedly by ingestion in milk we have an important source of evidence as to the transmissibility of the disease. On account of the extreme prevalence of human tubercular disease and the impossibility of tracing the source of infection, it is obvious that this evidence, unless carefully weighed, may easily lead us astray. It also is really little better than circumstantial. I have nothing to add to the able reviews of this part of our subject which have recently appeared.

FVIDENCE FROM EXPERIMENT.

Experimental evidence as to the identity or otherwise of human and bovine tuberculosis is not of recent date only, for we find that inoculation and feeding tests of tubercular products from man and from cattle had been made on various species of animals long before the bacterial nature of the disease was demonstrated. In an earlier report ${ }^{2}$ I have summarized the records of such of these experiments as admitted of comparison. Although these earlier infection tests were seldom designedly comparative, they nevertheless, when collectively reviewed, enabled us to recognize the same differences in the effects of the human and bovine virus which have recently been more clearly demonstrated by comparative infection tests with cultures. Nevertheless, the interpretation by different investigators of the results obtained in the past, has been no less contradictory than at the present day.

Such investigators as Chauveau, ${ }^{3} \mathrm{Klebs}^{4}{ }^{4}$ Crookshank $^{5}$ and others who have infected cattle with human tubercular virus have expressed the opinion that the resulting lesions are identical, anatomically and histologically, 
with those of Perlsucht-the naturally acquired cattle disease.

Creighton, ${ }^{6}$ Pütz, ${ }^{7}$ Klein and Gibbes ${ }^{8}$ are among those who, from similar experiments, arrived at the opposite conclusion; namely, that there was not only a difference in virulence of human and bovine tubercle when inoculated in cattle, but a difference also in the kind (as well as degree) of the resulting lesions.

The structure of the new formations induced by such inoculations in different species of animals, can not well be studied apart from the extent of these lesions; consequently, in reviewing the more recent work in this line I shall consider these two features together.

Up to the date of Koch's memorable discovery of the bacilli of tuberculosis, apparently identical in man and cattle, there was, as we have seen, a difference of opinion among pathologists as to the identity of the disease in the two species. It had been repeatedly demonstrated, for instance, that the bovine tubercular virus produced by inoculation in the rabbit more extensive lesions than sputa derived from human consumptives. Furthermore, a critical study of infection tests made by different authors, such of them as admit of comparison, will show that this higher virulence of the bovine tubercle was exhibited also toward other animal species, and more especially toward cattle.

This fact not being clearly brought out by comparative tests made by any one investigator did not receive due recognition. Pütz (1884), however, as a result of his researches, makes the following statement: "A susceptibility of cattle for the tuberculosis poison of man, and of the latter for the Perlsucht poison of cattle is not shown by any observations which are entirely free from objections." In his own experiments the results were always negative in bringing about in calves a typical Perlsucht with human tubercular material, but positive in producing a local development of tubercles containing the specific bacilli-"lesions no more representative of the typical tuberculosis of cattle than are the varcination pustules representative of variola proper."

Against this view may be placed that of Chauveau (1891), another investigator, who on several occasions had made comparative infeetion tests on cattle, and claims for his experiments the first complete experimental demonstration of the identity of the human and bovine disease. He states that "human tubercular virus reacts towards the bovine species exactly like tubercular virus coming from cattle."

With the discovery of the bacilli identical in their staining reactions in human and bovine tubercle, similar in cultural features, and in their effects on the guineapig, the etiologic identity of human tuberculosis and Perlsucht of cattle was generally conceded by pathologists, practically all writers on tuberculosis emphasizing more or less the dangers of human infection from the use of the milk of tuberculous cows. On the other hand, veterinary athors have generally assumed that the human consumptive may be a source of infection for the cow stable. It was with the view of testing this latter proposition that my own experiments in feeding calves with the expectorations of human consumptives were begun in 1896. In the same year was published the first report of Theobald Smith's inoculation experiments on cattle, ${ }^{\theta}$ the earliest comparative tests which had been made with cultures.

Frothingham $^{10}$ (1897) made communication of his inoculation experiments on calves with tubercle bacilli cultures of human origin. In the following year a further report ${ }^{11}$ was made by Smith of more extensive experiments undertaken in conjunction with the Massachusetts cattle commissioners.

Although my own sputum infection experiments for the Arkansas Agricultural Station were commenced independently, I am glad to admit that my later tests with cultures were modified, if not suggested, by those of Theobald Smith, to whom undoubtedly belongs the priority in this as in many other important lines of investigation and achievement in comparative pathology. Referring briefly to these experiments of my own, they were undertaken in order to test, as I have said, the possibility or likelihood of tuberculosis of cattle, and especially of dairy cows, having its source in the expectorations of consumptive attendants. I need not emphasize the vast importance of exact knowledge on this question from the standpoint of agriculture as much as from that of public health.

My experiments included (1) feeding tests on cattle with human tubercular sputum, (2) comparative inoculation tests on cattle with human and bovine tubercle respectively, and (3) similar inoculation tests with artificial cultures of tubercle bacilli from these two sources on cattle, sheep and swine. About the same time Pearson and Ravenel, of the University of Pennsylvania, were engaged in similar investigations, their infection tests also embracing a number of different species of the domesticated animals. The report of this work has lately been published. ${ }^{12}$

Koch and Schütz of Berlin have furnished the latest contribution to this line of investigation in the address of the former ${ }^{13}$ at the British Tuberculosis Congress of last year (1901). Although no new facts were brought to light by their experiments, the results of which were merely confirmatory of those previously obtained by American investigators, this address, as is well known, attracted world-wide attention on account of the great scientific reputation of the author and the somewhat extreme interpretation which he placed on the results obtained.

During the last year numerous other experimenters have been stimulated to a repetition of these inoculation tests on cattle with human tubercle, and even human martyrs have offered themselves, and been accepted by over-sanguine investigators, for inoculations from the bovine. Already we read of results: Calves have been successfully killed by sputum inoculation and human beings successfully infected (and cured) after inoculations from cattle; but we may well postpone any review of the work of these over-hasty experimenters until their enthusiasm moderates.

The more extensive and accurate of these recent experiments, namely, in order of publication, those of Smith, Dinwiddie, Pearson and Ravenel, Koch and Schütz, although not all co-extensive in design, have been practically in agreement in their results. It is therefore unnecessary to review each of them separately, and the summary which I here present is mainly the results obtained in my own experiments.

Inoculation of artificial cultures of the bacilli of bovine tuberculosis into the peritoneal cavity induces in calves, sheep and swine a rapidly progressive disease, early extension to the thoracic organs and death within a few months. Cultures of human origin (sputum) similarly inoculated, occasion in cattle a local eruption of tubercles over the serosa, especially abundant on the free omentum and in the sulci between the coils of the large gut. A tuberculin reaction may be obtained two weeks after inoculation. Sometimes no reaction is ob- 
tained, and in all my cases this reaction was absent four to five months after inoculation. The initial lesions are similar in kind to those produced by the bovine germ, the difference showing itself later by retrogressive instead of progressive changes. If killed within a few months there may be found in addition to the tubercles mentioned above, and now already calcareous, a greater or less formation of pendant fibrous fringes and small papular or pedunculated fibrous nodules outgrowing from various parts of the serous coating of the viscera. The abdominal lymph nodes may or may not be involved. If kept for a year or more before dissection all traces of earlier changes may. have disappeared. This, at least, has been my experience, and I have seen no impairment of health in cattle as a result of such infection from the human. I should not, however, dispute the possibility of inducing fatal disease in these animals by such inoculations. If the initial changes above described be so excessive as to impair the recuperative power of the tissues or their resistance to further invasion, the animal may succumb in a short time, either from the magnitude of the local changes or from unchecked generalization of the bacilli. Very young or feeble expcrimental animals, unhygienic surroundings, intravenous inoculations or intraperitoneal inoculations of impure cultures, such as we find in sputum, are the conditions on which we might suppose such a result to depend.

In sheep, human tubercle bacilli produce similar results as in cattle, but the local lesions are more extensive and more persistent. Pigs exhibit a somewhat greater susceptibility and may contract a progressive disease from such inoculations.

Generalization of the bacilli by way of the blood stream occurs in cattle, sheep and pigs, with formations of incipient miliary tubercles, which later become abortive in development, and are found on dissection as minute white points throughout the lung or as calcareous seed-like encapsuled granules in lung or spleen. On the other hand, if in pigs there is a pre-existent or intercurrent pulmonary disease generalization is followed by changes similar to those induced by the bovine germprogressive miliary tubercles with enormous multiplication of tubercle bacilli in the lung tissue or its morbid secretions. Inoculation of sputum instead of pure cultures may act in a similar manner by producing a mixed infection.

\section{BIOLOGIC CHARACTERS OF THE BACILLI.}

The remarks which I have to make under this heading are mainly derived from notes of rather hurried observations made during the progress of the inoculation experiments described in Bulletins $5 \%$ and 63 of the Arkansas Agricultural Experiment Station. They are somewhat fragmentary, but on the whole are in close agreement with the facts as observed and more fully described by Ravenel.

Tubercle bacilli, as has been frequently noted, present considerable variation in morphology under different conditions of growth. In sputum they may be long or short, straight or bent, granular or homogeneous in staining. A similar variability occurs in the bovine germ, and hence comparison is less exact than in species of more constant morphology. In general, bovine tubercle bacilli are shorter than those of man. In the caseous masses of tubercular lymph nodes in cattle the bacilli are usually rare, but occasionally I have found isolated collections of such material in which the bacilli were present in large numbers. In such of these speci- mens as I have seen the bacilli are uniformly short, granular or solid in staining, and not thicker than the bacilli of sputum. In ulcerating lung disease of cattle, as we have seen. this peculiarity in morphology is not present, the bacilli in such cases being undistinguishable from those of human sputum. I have not as yet had the opportunity of examining a sufficient number of specimens of bovine tubercle to venture an opinion as to the conditions on which this peculiarity in morphology, the short socalled bovine type, depends, but preserved specimens in my collection-cover-glass preparations-from other animals which have been infected with both the human and bovine bacilli, illustrate the following facts:

In the pig and sheep which have died from inoculation with bovine tubercle bacilli, the short form is not preserved. Smear preparations from the incised lung in both these species, the disease being generalized, show tubercle bacilli in great abundance and of the usual sputum conformation. In the pig, where I have also under certain conditions (a pre-existent swine plague pneumonia, or crude sputum inoculations) obtained a fatal generalized tuberculosis by inoculation with the human germ, the morphology of the bacilli is similar to that of bovine tuberculosis in the same animal.

In the rabbit and guinea-pig inoculated with bovine cultures exhibiting the short form, the results have been variable. I have slides in my collection in which the short type of bacilli can be recognized in smear preparations from these animals, while in others longer and medium forms predominate.

As to the form which sputum tubercle bacilli assume when grown in the organism of cattle I have unfortunately made no observations. In sheep and pigs the usual sputum form is maintained. In cattle the lesions resulting from such inoculations have been so slight and their contained bacilli so rare that no exact comparison is possible from any preparations in my possession.

In artificial cultures the bovine germ maintains the short type with more constancy. This has been especially observed by Smith in growing the bacilli on pasteurized dog serum. On the medium which $I$ have usually employed-glycerinized beef serum-although the short form usually predominates, the distinction between these and cultures from the human is not always appreciable. Bovine cultures are more difficult to start and generally make a feebler growth than human. The naked-eye appearances depend chiefly, I think, on the scantiness or luxuriance of the culture. On Löfller's medium I have noticed that the human germ assumes the short form of the bovine.

An explanation of the conditions on which this variability depends is not possible with our present knowledge. It is manifest, however, that the source of the bacilli in the sputum of the human consumptivehuman or bovine - is not to be determined by their morphology.

In comparing the animal tuberculoses with that of man, we have limited our discussion to the bovine disease. Tuberculosis, however, occurs in other mammalian species. It is not rare in pigs and is occasionally found in horses, dogs and cats, while a very few instances are on record of its occurrence in sheep and goats. Wild animals in the state of nature are, I believe, entirely exempt, but under confinement in cages some species are peculiarly liable to fall victims to this disease. Are we justified, in view of these facts, in assuming the existence of races of tubercle bacilli peculiar to each zoölogic species in which tuberculosis naturally 
occurs? The very rarity of the discase in most of thes? species precludes this idea.

In pigs the source of infection can be traced to ingestion of the milk of tuberculous cows. Cats and dogs are infected in the same manner, or from close association with their consumptive owners. Although under conditions of close confinement, as in menageries, we may well believe that transference of the disease may occur between animals of the same species, the original source of infection is probably in all cases extraneouseither from mankind or from cattle.

In the case of the human and the bovine disease the conditions are different. In both these species tuberculosis is an cxtremely common affection. Contagion in cattle tuberculosis can frequently be traced without difficulty, and its source is in other cattle similarly diseased. We have seen from the experiments just described that a human origin of the cattle disease is hardly conceivable. If it occurs at all it must be under most exceptionally rare conditions. On the other hand, we may assert, I think without begging the question, that in most cases at least human tubercular disease has its origin so far as contagion is concerned in other similarly diseased human beings. We have here, then, the conditions on which depend the evolution of stable racial characteristics in the bacteria; namely, long-continued invariability of environment. We find, therefore, in these facts a theoretic justification in assuming, at least hypothetically, the existence of two races of mammalian tubercle bacilli, the human and the bovine. Practically this assumption is supported by the facts in reference to differences of morphology and pathogenic virulence just discussed.

\section{DEDUCTIONS.}

From the foregoing data we have now presented to us the task of deciding whether the experimental evidence tends to support or to refute the view that human consumption may at times have its origin by contagion from cattle. I can best introduce my own views on this matter by a quotation from my first report on tuberculosis, already referred to: "It can not be said that the facts here presented remove the question in any degree out of the domain of speculation. They really furnish more food for theorizing, and may either diminish or increase our suspicion of dairy products according to the process of reasoning employed. It may be argued, for instance, since bovine tubercle bacilli have been shown to be equally as virulent as those of human origin for all animals tested, and more virulent for some, the presumption is that they are more virulent for mankind also. Contrarywise it may be said that human tubercle bacilli being shown to be less adapted for growth in cattle than the bacilli of bovine origin, there is reason for believing that the converse may also be true." "If the bovine bacillus be regarded as merely a more highly virulent modification of the human variety, this should be shown by the greater gravity of the lesions produced by comparative inoculation experiments on all species of susceptible animals. If, however, there is a selective feature shown by such inoculations, the excess of virulence of the bovine variety being manifested only toward certain species of animals, some support would be gained for the theory of a distinction of races other than that founded on a mere difference in degree of pathogenic activity."

The tests necessary to determine this latter point have since then been made and the records are contained in my second report ${ }^{14}$ on tuberculosis (1900).

The bovine tubercle bacillus has been shown by these experiments to possess an indiscriminate and not a selective excess of virulence over the human specimen. Similar results have been obtained by Pearson and Ravenel of Pennsylvania and by Foch and Schütz of Berlin.

In a purely speculative question it is a matter of everyday experience that the same data may lead to entirely different deductions. Professor Koch, whose attainments and achievements in bacteriology we all respect, has deduced the inference, largely, as we infer, from his experimental work on animals, that bovine tuberculosis can constitute no factor in the initiation of tuberculosis of man. Few of us, I think. will be able to follow this process of reasoning. Whether or not his view is correct it can hardly be said that it derives any support from the purely experimental evidence. I think there is a wide field for investigation yet to be covered before we as sanitarians can feel justified in recommending the discontinuance of those safeguards against infection from tuberculous dairy products which an unwilling public are just beginning to appreciate.

$$
\text { BIBLIOGRAPHY. }
$$

1. Tuberculosis, the True Malaria. Paper presented at the annual meeting of the Arkansas Medical Society, Little Rock, May 13,1902 .

2. Bull. No. 57, Ark. Agr. Expt. Station, 1899

3. Bul. de l'Acad. de Med., 1868 , p. 1007 ; also Rec. de Med. Veter., 1869 , p. 202 ; also, Assoc. Franç. pour l'Ávance de Sc. Compt. Rend., 1873, p. 717 ; also Congrès pour l'Etude de la Tuberculose, $1891, p .51$.

4. Archiv für path. Anat. u. Physiol., Bd. 49, 1870, 1871

5. Trans. Pathol. Society, vol. xlii, 1891, p. 330 (Text-Book of Bacterlology, 4th Ed., 1897, p. 390.)

6. Jour. Anat. and Physiol., 1880-1881, vol. xv, p. 1.

7. Versammlung deutsche Naturforscher in Éisenbach, 1882 Wiener Mediz. Blaetter, No. 41 ; Deutsche Med. Ztg., 1884, i, S. 557. 8. Rep. Med, loc. Gov. Bd., London, 1883-4-5.

9. Trans. Assoc. Amer. Physicians, xi, 1896.

10. Rep. Mass. Cattle Comm'rs, 1897 , p. 50.

11. Jour. Exp. Med.. iii, Nos. 4, 5, 1898, p. 451

12. Univ. Penn. Med. Bul., September, 1901

13. British Congress on Tuberculosis, 1901.

\section{DISCUSSION*}

ON PAPERS OF DRS. KNOPF, APPEL, CARRINGTON, BONNEY, STUBBERT, SALMON AND DINWIDDIE.

Dr. M. P. Ravenel, Philadelphia-We can hardly speak of this question without bringing in Koch. As you are aware, he laid down two propositions: First, that human tuberculosis differs from bovine and can not be transmitted to cattle; and second, that if man is susceptible to infection from cattle at all it is so rare an occurrence as to make precautions unnecessary. Now the first of these propositions is capable of being subjected to experimental proof. You will remember that Koch, in the experiments conducted by Shütz and himself, did not succeed in producing bovine tuberculosis with the human bacillus. In my opinion the conclusions of Koch were based on an insufficient number of experiments. At the laboratory of the State Live Stock Sanitary Board of Pennsylvania, we have succeeded in doing what he failed to do, and $I$ have placed in the Scientific Exhibit specimens of animals that were infected with the human disease. One shows the liver and diaphragm to be affected. This was the result of an attempt we made at obtaining immunization; we got a result we did not wish for, but one that is of importance in establishing the fact that human tuberculosis can be communicated to animals. We must acknowledge that bovine animals, as a rule, show a high degree of resistance to infection from man.

Generally speaking, the pathogenic power of the bovine tubercle bacillus is considerably greater than the human for all experimental animals. Of course, this does not prove that bovine tuberculosis can be transmitted to man, but this can be proved without any trouble. We can not, it is true, carry out experimental tests as we do on animals, but there is a large

* The papers of this Symposium on Tuberculosls which have already appeared will be found in the following issues: Dr. Knopf, November 22 and 29 ; Dr. Appel, November 29 ; Drs. Carrington, Bonney and Stubbert, December 6 .

The symposium will be completed later by the following paper and its discussion:

Sanitary Measures for the Prevention of Tuberculosis in New York City, and Their Results. Herman M. Biggs, M.D., Newo York City. 
amount of evidence available of accidental inoculation of man from cattle. It is well known that the usual tendency of skin infections is to remain localized, but in the case of infection from bovine tuberculosis it has been shown that it may become systemic, a number of cases being on record in which the disease became general following a local infection and ended in death. Four cases of accidental inoculation of man by the bovine tubercle bacillus have been reported to the Pathological Society of Philadelphia, accompanied in three instances by the exhibition of the specimens. Two other cases have been reported by Dr. Hartzell of the University of Pennsylvania, in which, though the bovine origin of the infection was not absolutely proved, it was highly probable. They occurred in strong, healthy men who were employed in cleaning cattle cars. They contracted skin tuberculosis following slight injuries to the hand by broken timbers. In one the disease spread to the lungs and other internal organs, and the patient dicd within a year. The other recovered under local treatment. Where a man has a local tuberculous affection starting on the back of his hand, and exterding to the lung and other organs, I think we are justified in saying that the disease has come from the local infection. I admit freely that there are gaps in our evidence, but it is at least as clear and convincing as that we accept daily and without question when the source of a case of scarlet fever or typhoid fever is under examination. The fact is, positive demonstration of direct transmission of most diseases is extremely rare.

Since interest has been revived in this subject by Koch's paper, in which he repudiated his former views as to the intercommunicability of human and bovine tuberculosis, numerous reports have appeared of cases of the kind referred to, in which persons were accidentally inoculated. In Berlin alone five eases have recently been reported, all occurring in men employed in slaughter houses, and commencing in wounds about the hands.

It would seem that instances of infection through wounds are not as uncommon as has been supposed heretofore. Their value has been questioned on the ground that inoculations do not always correspond to natural infections. I do not pretend that such cases settle the whole question definitcly, but they do prove beyond question that there is no peculiar quality in the tissues of man which makes him an unfit soil for the bovine tubercle bacillus. Furthermore, they afford us means of comparison with similar cases in which the invading organism is of human origin, wounds of the hand while operating on tuberculous cadavers, or in cleaning cuspidors used by phthisical persons, being not uncommon. I have been at some pains to study the reports of such infections, and feel bound to conclude that the bovine bacillus is at least as virulent as the human for man when introduced in such manner. Wt know from experimental as well as clinical evidence that the skin is the tissue most unfavorable to the growth of the tubercle bacillus. If, then, the bovine bacillus can successfully invade the skin and multiply there, with the production of characteristic changes, it seems we are fully justified in believing that organs and tissues that are known to offer favorable soil to the human bacillus will also prove favorable to the bovine organism. On the other hand, I know of no animal which is susceptible to the human bacillus yet immune to the bovine. Birds are equally refractory to both, a small proportion of those inoculated or fed with either succumbing to tuberculosis.

The next point in this discussion is to controvert the idea that a food infection must be looked for solely in the intestine. By so doing you leave out of consideration the pharynx and tonsils, both of which are important avenues of infection.

In our work at the laboratory of the State Live Stock Sanitary Board we have often been struck by the extensive in. volvement of the lungs in animals infected by feeding, and the slight injury of the intestines. Indeed, in some animals it has been impossible to detect any involvement of the intestine at all.

In a cow which was fed with bovine tubercular material, both lungs were involved and had cavities in them. The bronchial glands were enlarged and showed small nodules. The mediastinal glands were enlarged and softened, but showed no nodul's. The mesenteric glands were normal, and no lesion of the intestinal wall could be found.

In a monkey fed with a pure culture obtained from the mesenteric gland of a child both lungs were involved throughout and to a much greater extent than the abdominal organs. ine bronchial glands were enlarged and caseous. In the intestine only one point of injury could be found, and most of the mesenteric glands were normal in size, and showed a few nodules $1 \mathrm{~mm}$. in diameter. Three glands associated with the upper intestine were much enlarged and showed cascation, though not more advanced than what was seen in the bronchial glands.

The specimens from the monkey illustrate another important point, namely, the occurrence of coincident infection througls the upper part of the digestive tract and the intestine. If this animal had been examined without the knowledge of its history it would almost certainly have been put down as one of those cases in which the avenue of infection could not be positively determined. The lungs show much more extensive disease than any of the abdominal organs, and the bronchial glands show caseation, as well as some of the mesenteric, and to an cqual degree. This monkey was tested with tuberculin before the experiment began, and was in perfect health when the feeding with tubercle bacilli commenced. The key to the situation lies in the enlargement and caseation of the cervical lymphatic glands, indicating an infection through the tonsils or some neighboring part of the upper digestive tract, though no lesion could be detected. We have here an undoubted food tuberculosis, yet the respiratory tract shows the chief involvement. Does not the same thing occur also in children, and more often than we suspect?

In another experiment, four swine were fed with pure cultures of tubercle bacillus. In three of the four there was extensive destruction of the tonsillar tissues, and in all widespread involvement of the lungs and other organs, while in one only could any trace of injury to the intestine be found. We are surely justified in believing that similar things occur in children. If the milk with which a ehild is fed happens to contain tubercle bacilli, they naturally find lodgment in the tonsils, owing to the frequency of feeding and the structure of the tonsils.

The number of cases in which infection can be directly traced to the consumption of tuberculous milk is not large, and almost all of them are open to some criticism from the fact that all other sources of infection can not be positively excluded. We can not shut children up and feed them with tuberculous milk by way of an experiment; but the evidence furnished in many of the cases which have been reported is so clear that, as Nocard has well said, "It has almost the value of an ex. periment," and there is no room to doubt that such infection does occur. In our experimental work we have had convincing proof of this. We have isolated from the mesenteric glands of two children who died of tuberculosis cultures of the tubercle bacillus which are virulent for cattle. From one of these children we obtained a culture which was extremely virulent, causing death in a cow 6 years old in 18 days, with extensive and typical lesions. Now it follows that one of two propositions must as a consequence be admitted: Either we have found a human tubercle bacillus having a pathogenic power for cattle quite as great as any bovine germ, or else we have found in the mesenteric gland of a child the bovine tubercle bacillus. In other words, the human tubercle bacillus is at times just as virulent as the bovine, or else the child has been killed by infection derived from bovine sources. For many reasons $I$ am inclined to believe that the latter is the true explanation.

In ehildren tuberculosis shows a marked tendency to become generalized, and it is in them that we have most reason to fear infection by food, their diet consisting so largely of cow's milk.

To sum up: Of one thing I have no doubt whatever, and that is that bovine tuberculosis constitutes a grave source of danger to human health. No doubt there may have been exaggeration in the past as to the number of cases of tuberculosis that were directly traceable to the dairy; but even if this exaggeration had been ten times as great, it does not justify 
the attempt now being made to blind ourselves to it as a source of infection. A danger is best guarded against when best recognized; and while I do not at all deny that there are many other sources of infection, I say that in tuberculosis of cattle we have something that must be guarded against, and that it is our duty to educate the public on this subject with a view to the adoption of eviry possible precaution to prevent the spread of the disease by this as well as by other means.

DR. S. A. KNopr. New York-I was one of those who had an opportunity of listening to Koch's address in London, and 1 shall never forget the sensation it caused. The very moment he sat down, however, the venerable Lister got up to oppose the views that had been advanced, and the majority of the other speakers were very much in favor of what Lister said, or of at least suspending judgment until the matter had been more fully investigated. As the result of experiments that have been made since by Dr. Ravenel and others I believe that we are abundantly justified in saying that the two kinds of tuberculosis are interchangrable. For my own part, I have not made any laboratory investigations myself to prove or disprove Koch's theory. If, however, any one in authority came to me with strong laboratory evidence that bovine tuberculosis could not be transmitted, I still would have great hesitation, after what $I$ have observed clinically, to give a child milk from a tuberculous cow. Most of us, I think, are pretty well convinced that bovine tuberculosis can be transmitted to human beings, and that every conceivable prccaution should be taken to safeguard the public from this source of infection.

But there are some points in regard to which I can not agree with Dr. Appel. He spoke, for example, of patients being "clinically cured," and by way of explaining what he meant, he said he was in the habit of injecting tuberculin to see if the disease was eradicated. That is a practice against which $I$ must protest. How many of us after being pronounced cured would care to submit to a tuberculin test? For my part I hesitate to use tuberculin even for diagnostic purposes. I do not think it at all necessary or desirable that we should subject patients who have been apparently cured to such a dangerous experiment. I have in mind a case that should act as a warning to all of us-that of a prosperous young merchant about whose symptoms there was some doubt, and who was given a small dose of tuberculin, resulting in general tuberculosis and death within a few weeks. As to the supposed therapeutic value of tuberculin, I will say that $\Upsilon$ shall con tinue to refrain from its use until we know a great deal more about its action than we do now. I repeat, that I consider it a very dangerous experiment to use it on a patient who is thought to be cured. I try to keep abreast of all the literature on the subject, but I must say I never read of any one else doing such a thing. As to the description of the sanatorium, it was in all respects very interesting, but I would like to ask Dr. Appel how far apart the beds are.

DR. APPEL-They have 4,000 feet of air space and are some three or four feet apart.

Dr. KNOPF-I am afraid that three feet is hardly enough. Patients with dry coughs are apt to spread the disease through drop infection, and therefore $I$ would be in favor of having the'm further apart.

I have no doubt Dr. Bonney is well justified in all that he has said about the beneficial effects of the climate of Colorado, but the unfortunate thing is that we can not give change of climate to the majority of our tuberculous patients, however much we might desire to do so. We must treat the great majority of them at home, and until we realize that fact we will not make much progress in the solution of the tubcreulosis problem so far as the great masses are concerned.

Dr. Boardman Reed, Philadelphia-It seems clear to my mind that human tuberculosis can be transmitted to animals. That, I think, has been thoroughly proved. The difficulty is as to the converse proposition-the transmissibility of bovine tuberculosis to man. I am convinced that that likewise has been pretty well established. Koch says it has not been proved positively, and no doubt it is not easy to do so. But there is one way by which it might be done, and that is to allow criminals condemned to death to be placed in the hands of responsible medical men for the purpose of experiment. Many of them would consent to take the chance of escaping from death. The governments of many of the states would no doubt grant the privilege if properly approached, and with a view thereto it might be well to have a motion passed on the subject if the section approves of the suggestion. There is ont other point to which I would like to allude. In Dr. Stubbert's very able and practical papur he spoke of the methods of treatment in cases of dilated stomach. That is a matter of very great importance and one that requires the exercise of great caution. It is espccially necessary so to regulate and limit lavage when this is required as to avoid washing out any portions of partly digested food. The best time to practice lavage in these, as in most other cases of gastric dilatation, is in the morning before briakfast. 'Tuberculous patients are generally ill nourished and lavage is often badly borne by them, especially when frequently done or too soon after eating.

Dr. C. L. Minor, Asheville, N. C.-I believe that our sanatoria will not be complete until the r'rench idea is carried out, and each of them has a convalescent farm attached to it. If people go back to the slums they will soon relapse into the condition they were in before. The only way to ensure their recovery being permanent is to afford th'm facilities for outdoor work for a time in a suitable climate, and this can best be accomplished by means of sanatoria farms, such as are now so common in France.

As to Dr. Knopf's paper, I have tried Japanese handkerchiefs and chcesecloth as sputum receivers for the patient while walking or away from home, but I am not satisfied with either or with the ordinary sputum flask, whose opening is far too small for cleanliness. Something better is required, and down in the exhibit hall $\mathrm{I}$ saw a contrivance in the shape of a telescoping cigar case of paper that impressed me very favorably. Something in that line or like that shown by Dr. Appel is called for. One point in Dr. Appel's paper struck me very forcibly: the tendency to over-exercise. The general practitioner is too apt to tell the patient on sending him away to Colorado or elsewhere to take all the exercise he can. We ought, on the contrary, to warn our patients against fatiguing themselves with too much exercise. They should take all the rest they can. That is the first consideration. They can not do themselves any harm by over-rest, but they can kill themselves with over-exercise.

DR. A. RoBIN, Newark-There have been in literature numerous references to tuberculin, and I rise simply for the purpose of saying that $I$ do not see anything in it which would produce miliary tuberculosis. Were it so, the extensive use of tuberculin in the diagnosis of tuberculosis in cattle would result in many cases of generalized tuberculosis. I thought the consensus of opinion in the profession was that the tuberculin test was a perfectly harmless procedure. I do not see any objection whatever to using it for diagnostic purposes.

Dr. H. D. Holton, Brattleboro, Vt.-I would like to ask Dr. Ravenel whether the symptoms of the disease he found in the autopsies on the children were in the pearl-form of lesion.

Dr. RAVENEL-You usually find them in large numbers clustered round nodules.

Dr. StubBert-As to the tuberculin test, while I do not feel so strongly as Dr. Knopf, I am bound to say that I have never been inclined to use it for diagnostic purposes, and I would be very much afraid of lighting into renewed activity an arrested tuberculous focus if I were to use it to see whether a cure had been effected. Besides, it is well to remember that even for diagnostic purposes it can not be implicity relied on, because we can obtain a reaction from syphilis as well as tuberculosis, and in a given case it is never certain which it is. As to the arrangement of sanatoria, I believe that separate buildings are better than any dormitory. I agree with Dr. Reed, that we can not exercise too much care in connection with the treatment of the stomach. As to the $x$-rays, I have simply to add that I think they possess great possibilities of usefulness. Finally, to say what may seem hardhearted-we have to fight public sentiment and abandon the policy of wasting money building sanatoria for incurable cases. If we are to make headway against the disease, we must concentrate our attention to a much greater extent on cases that can be cured. Unless we do this we will find our- 
selves in the position of the man who writes on sand at the seashore, all of whose words are washed out by the incoming waves as fast as he writes them. The fact that the board of health in New York City has been able to reduce the number of tuberculous cases in the past three years 35 per cent. by simply educating, in a limited way, the tenement dwellers, makes it reasonable to suppose that, if we would concentrate our efforts on curable cases of tuberculosis, we could almost totally eradicate this dread disease.

Dr. H. M. Bracken, Minneapolis-Dr. Knopf referred in his paper to some states where tuberculous subjects are taken care of if they are criminal or insane, but not otherwise. One of the states thus referred to was Minnesota. Now I think we should guard against speaking sneeringly about any efforts that are being made to check this dreadful disease. We must take conditions as we find them, and remember that there may be circumstances to account for apparent anomalies of the kind referred to. It by no means follows that in Minnesota and other places where provision has been made for the care of the criminal and insane we are indifferent to the welfare of those who do not belong to either class. It simply means that we have got those classes segregated in such a way that they can more easily receive attention. Efforts are being made in all the state institutions of Minnesota to look after and improve the health of the inmates. Reference was also made to the ease with which legislation can be obtained for the suppression of bovine as compared with human tuberculosis. I am afraid there is too much ground for this criticism.

DR. R. C. Moore, Omaha-We have heard some excellent papers on the sanatorium treatment of tuberculosis, but all of them come from gentlemen who have charge of institutions that are situated in the most favorable climatic conditions. I think it is unfortunate that we have not heard from any of those who have had experience in institutions not so favorably situated. A little to the east of us is one of the most liberally conducted institutions of the kind in the United States. I refer to the Massachusetts State Sanatorium at Rutland. The report published by the managers of that in stitution gives results quite as good as those obtained by Dr. AppeI and Dr. Carrington under the most favorable climatic conditions. All of you know that New England has one of the bleakest and most uncomfortable climates to be found in the country, and if such excellent results can be obtained by sanatorium treatment there, it is a matter of great importance, for it shows that it will no longer be necessary for us to transport our patients thousands of miles in order to get salubrious climiates. I wish there were some one present who had had practical experience in some of these eastern sanatoria, for it is practically impossible for us to send the majority of our patients to those distant places with fine climates. Out of every hundred tuberculous patients there are sure to be more than ninety who are unable to leave their homes. These are the people that must be provided for, and there is only one way by which this can be done, namely, by the states establishing sanatoria in the immediate vicinity. There should be an institution of the kind in every district where there is a sufficient population to demand it.

Dr. S. G. Bonney, Denver-The last speaker has claimed more for the sanatoria in the east than the gentlemen who conduct them have ever cared to claim. We should remember that their results and conditions are not comparable in any way with those submitted in the papers that have been read to-day. There is a vast difference in the character of the cases. Dr. Appel and Dr. Carrington have to take the cases as they come-many, perhaps the majority of them, broken-down old soldiers and sailors, not a few of them with alcoholic histories. We have been given some very favorable reports as to what can be done in these cases by means of sanatorium treatment under favorable elimatic conditions. At some of the eastern institutions they do not take the cases as they come, but are permitted to select them. Even those who do gain admission are not chosen in accordance with their order of application, but according to the more or less favorable condition. There is frequently a long waiting list, and those are selected from it whose symptoms give promise of the most speedy recovery. If Dr. Moore will read the reports carefully he will see the necessity for distinguishing between the cases and the results, which are not properly subject to comparisor.

I think it was Dr. Appel who spoke of breathing exercises, and said that after three years in the west his own chest measurement had increased three inches. I am not prepared to admit that this was entirely due to breathing exercises. Some time ago a troop of soldiers went to Fort Logan from near the sea level. Their measurements were taken at intervals, and after a time, at an elevation of 5,000 feet, it was found that they showed an average expansion of two inches. So it does not follow that the increase in measurement whicb the Doctor tells us he has obtained is due to the breathing exercises. Personally, I am opposed to any breathing exercises for consumptives at high altitudes. I am opposed in general to any effort to compress the lungs by means of nitrogen gas or otherwise, and I am also opposed to all measures looking to their expansion. We are too apt to lose sight of the fact that it is the tuberculous individual rather than the tuberculous lung that we are treating. We should keep our patients quiet and get them into the way of leading a life of inactivity and indolence for the time being.

Another point I would criticise was the system of classification referred to by Dr. Appel. As I understand him, he divides his cases into three classes according to their stage as shown by (1) infiltration, (2) consolidation, (3) caseation I think it is fallacious for us to make any prognostic elassification according to physical signs. We may compare a dozen cases and be utterly unable to form any prognosis according to physical signs per se. Other factors must be taken into consideration, and they are so important as to make any such attempt at classification of little if any use, beside being misleading.

Dr. Apper-As to Dr. Knopf's objection to the use of tuberculin as a test of cure, I must say that in an extensive use I have never observed any harm resulting from it in the way we use it. We get it from Dr. Baldwin, who prepares it at Trudeau's laboratory at Saranac Lake, where it has been used for years, and I am assured without any deleterious effects. When the diagnosis is doubtful we use it in conjunction with other means to establish the presence or absence of the disease. We adopt every means to make a diagnosis, and I believe the tuberculin test equally as satisfactory as is the Widal test in typhoid fever. The classifications made in my paper were not intended to be scientific classifications for the study of the disease. The first divides the cases as we practically separate patients in their dormitories, those who have no bacilli being in separate dormitories, while the permanently febrile are kept in the infirmary. The other class consists of those with bacilli who have no fever, some having been temporarily febrile. The other classification was made for the purpose of charting the cases and to compare our results with others. I can not concur in the opinion of Dr. Stubbert as to the prognostic value of the position of the heart. In many cases its position is changed by contracted adhesions. As to breathing exercises, I never saw any bad effects from them. Of course, they are made gently and the patients are cautioned not to make any violent muscular effort. $\mathrm{Dr}$. Bonney has suggested that the inereased girth of the chest is due to the altitude and not to these exercises. In my own case I served for many years in high altitudes before, and the girth of my chest had not increased. In the experiment with the soldiers, which he quoted, they are compelled to regularly take prescribed setting-up exercises, which are intended to increase the size of the chest.

Dr. KNopF-It is quite true, as has been stated, that I spoke of some states taking care of the insane and others of the criminal classes while they did nothing for the sane and the non-criminal. I did not wish to imply that it was wrong for those states to care for the classes referred to. I only wished to emphasize the necessity of likewise taking care of those who come under neither category. Dr. Appel seems to 
have misunderstood my remarks about dormitories. I think there is a danger of patients expelling small particles of bacilliferous saliva during dry cough, and if the beds are too close together drop infection is possible. As to breathing exercises, I have taught them for years to my patients, and, as in Dr. Appel's case, the teaching led to an increase of $\mathrm{my}$ own chest expansion; but I only had New York air, while Dr. Appel had the clear, dust-free atmosphere of Fort Bayard.

Mr. Chairman: I move the adoption of the following resolution :

RESOLUTION ON TUBERCULOSIS.

Resolved, That in view of our knowledge that several European governments have established national governmental tuberculosis commissions, and recognizing the fact that tuberculosis is likewise with us a very prevalent and a preventable disease, the Section of Hygiene and Sanitary Science recommends that the American Med ical Association address an appeal requesting the proper federal authorities to create a United States Tuberculosis Commission for the study, investigation and prevention of tuberculosis in man and animals.

Dr. BENJAmin LEe, Philadelphia-I have much pleasure in seconding the motion. This question has now been raişed to the dignity of a National one. Until we had reasonable ground for believing that bovine and human tuberculosis are intercommunicable and that the condition is one which is, to a large extent, curable, we would not have been justified in calling on the National Government to interfere. I believe, however, that the time has now arrived when we can with propriety ask the American Medical Association to use its influence with the government to get such a commission established. It will not be creating a new precedent, for, acting in co-operation with the American Public Health Association, we have already induced the government to undertake the investigation of yellow fever and the possibility of its preven tion. It is true that in the case of the latter disease the conditions were such that the commercial instinct could be appealed to more easily. Yellow fever interfered with commerce over a considerable portion of the country, and therefore it was not difficult to get the ear of Congress. It is doubtful if we shall as readily succeed in our efforts in regard to tuberculosis, but, none the less, it is our duty to make the attempt.

(The motion was adopted and referred to the House of Delegates and referred back to the Section for elaboration. See The JourNal, June 21, 1902, p. 1658.)

\section{DERMATITIS REPENS.*}

M. B. HARTZELL, M.D.

Instructor In Dermatology in the University of Pennsylvania; Dermatologist to the Philadelphia Hospital. PHILADELPHIA.

In 1888 Crocker first called attention to a peculiar inflammation of the skin to which he gave the name dermatitis repens, his description of the disease being based on three cases, of which the following is a brief summary:

CASE 1.-The first case occurred in a young man who had had a portion of a finger amputated on account of an injury. The operation wound healed in normal fashion, but at its border a dermatitis, resembling in a general way an eczema rubrum, began which gradually spread up to the palm and over the fingers. The surface was denuded of its epidermis, was intensely red, oozing a clear fluid like sweat from numerous points around the sharply defined borders of the patch, which consisted of undermined epidermis elevated by fluid. The inflammation continued to extend for some months until it reached the elbow, the hand in the interval having recovered its normal condition. After trying applications of silver nitrate and copper sulphate without favorable results, recovery took place under continuous application of lactate of lead.

CASE 2.-The second patient was a weak, nervous woman, 28 years of age. The inflammation began on the flexor surface of the wrist with an eruption of red papules, which coalesced

* Read at the Fifty-third Annual Meeting of the American Medical Association, in the Section on Cutaneous Medicine and Surgery, and approved for publication by the Executive Committee :
Drs. W. T. Corlett, L. Duncan Bulkley and W. L. Baum. and discharged. It spread down the hand and up the arm, presenting a well-defined border covered with thick crusts; it ultimately reached the shoulder, spread across the back of the neck and down the other arm to the elbow, the older parts of the disease in the meantime healing. It was finally cured by cutting away the undermined epidermis and applying a 10 per cent. solution of permanganate of potash until it formed a crust.

CASE 3.-The third of these cases occurred in a young man, 19 years old, and was much milder than the two preceding. The disease began as an eruption of small blisters on the wrist one year before, spreading to the arm and hand until the whole palm and fingers, except the terminal phalanges, were involved. Recovery took place in about three months.

In 1892 , in a short paper read at the International Dermatological Congress held at Vienna, Crocker again called attention to the malady, reporting two new cases and referring briefly to others which had been under his own observation or that of others. In this paper he describes the affection as "a spreading dermatitis starting from an injury to the skin . . . usually manifested by a free exudation of fluid in the form of vesicles, or a bulla, the result of which is the complete denudation of all the upper layers of the epidermis, leaving an intensely red surface oozing in numerous points. . . The disease spreads by direct extension, not by the formation of fresh foci either near or away from the original morbid area. It may extend over a part or the whole of a limb, or even over a large portion of the body, and is not accompanied by marked itching, burning or disturbance of the general health. Finally, although very rebellious to treatment, it ultimately yields to local remedies of an antiseptic character while internal remedies appear to have no effect." In all the cases except two the disease was preceded by and started from an injury of some kind, trifling or severe. The youngest patient in whom the affection was observed was a girl of 11, in whom it followed the running of a splinter under the nail; the oldest was a man, $87^{\circ}$ years of age, in whom the eruption appeared 48 hours after an injury to the left shoulder and arm. In 1896 Stowers reported a case as follow:

CAse 4.-A woman, 67 years old. Fourteen days after a confinement, when she was 29 years of age, a small "gathering" was noticed in the matrix of the right thumb-nail which was followed by loosening and detachment of the nail. This never healed according to the statement of the patient, small pustules appearing around it from time to time. A year later the left thumb became affected in a similar manner, and subsequently some of then fingers with the nails were involved at intervals of several months. At a much later period the toes began to be affected, the nails of which were lost. Severe burning pains, such as often accompany peripheral neuritis, were frequently present for long periods in the affected areas. Microscopic examination for the ringworm fungus gave a negative result.

Frèche has reported as "a trophoneurotic eruption of the extremities recalling dermatitis repens," the following case:

CASE 5.-A man, 48 years old, after some months of fatiguing labor, began to sufler from severe nocturnal pains starting in the nape of the neck and extending along the arms, accompanied by sensations of heat in the hands. Some four or five months after these pains had almost entirely ceased, a brownish patch appeared on the nail of the right thumb without any apparent injury, although there was a doubtful history of a prick with a thorn. Some days later redness and swelling appeared about the insertion of the nail, then pus, which loosened the nail and the epidermis. The disease gradually spread into the palm and the other nails became diseased; and at a later period analogous lesions showed themselves on the left thumb. All the palm of the right hand and the palmar surface 